\title{
Cognitive function is associated with impaired heart rate variability in ageing adults: the Irish longitudinal study on ageing wave one results
}

John Frewen, Ciaran Finucane, George M. Savva, Gerard Boyle, Robert F. Coen, 4 and Rose Anne Kenny,

\section{Abstract \\ Objective}

To examine the independent association between heart rate variability (HRV) and cognitive performance, in a nationally representative population study of older adults.

\section{Methods}

Cross-sectional analysis of wave 1 data from the Irish longitudinal study on ageing (TILDA) was performed. A subset of 4,763 participants who underwent ECG recording during resting and paced breathing periods were used for the analysis. HRV indices were divided into quintiles for comparison of values and cognitive performance was defined using the Montreal cognitive assessment (MOCA) score. Multivariate linear regression was used to model the association between cognition and different quintiles of each HRV index, after adjustment for covariates.

Results

The mean age was $61.7 \pm 8.3$ years and 2,618 (55\%) were female. Lower quintiles of SDNN ( $\mathrm{P}=0.01$-paced), $\mathrm{LF}(\mathrm{P}=0.001$-paced), and LF:HF ratio $(\mathrm{P}=$ 0.049-paced) were significantly associated with lower MOCA scores (during both recording periods), independent of confounders. Sub-domains of MOCA responsible for the relationship were predominantly memory recall and language.

\section{Interpretation}

Reduced HRV is significantly associated with lower cognitive performance at a population level in people aged 50 and older. This further strengthens the relationship between autonomic dysfunction and cognitive disorders.

Keywords

Autonomic function Heart rate variability Cognitive impairment Paced breathing Population studies

Introduction

Ageing is associated with a reduction in cognitive performance. The average incidence rate of mild cognitive impairment (MCI) is $12-15$ per 1,000 person years in persons aged 65 and older [2]. The conversion rate from MCI to dementia is $14 \%$ annually [30]. Predictors of conversion from MCI to dementia 
currently include cerebrospinal fluid biomarkers, neuropsychological tests and structural magnetic resonance imaging (MRI) morphometry $[8,11,42]$.

The ageing demographic shift will likely increase the global burden of cognitive disorders in the coming years. In light of this, there is an urgent need for interventional strategies targeting modifiable determinants of MCI, dementia and conversion from MCI to dementia. Factors associated with cognitive decline include age, lower education, lifestyle (lower physical activity, smoking and high alcohol consumption), genetic predisposition, metabolic dysregulation (obesity, impaired glucose tolerance, diabetes), cardiovascular disease (hypertension, atherosclerosis, stroke) and inflammatory markers, many of which are evidently modifiable $[13,16,36,39,45]$.

Many of these factors are also precursors to reduced heart rate variability (HRV), including smoking, high alcohol intake, obesity, diabetes, hypertension, and inflammation, $[5,12,31]$. This suggests that HRV and cognition have common risk factors. HRV is a marker of cardiac autonomic function, and involves the interaction of activity from the sympathetic (SNS) and parasympathetic nervous systems (PNS). Emerging evidence suggests an association between cognitive impairment and autonomic dysfunction. This relationship may be bidirectional with autonomic dysfunction causing systemic hypotension that leads to cognitive impairment [27], while neurodegenerative processes influence autonomic pathways, causing autonomic dysfunction [19].

Studies investigating the association between HRV and cognitive function have to date reported conflicting results $[3,15]$, however, none have been conducted in large population based samples. The aim of this study was to investigate the independent association between HRV and global cognitive function, in a nationally representative sample of older adults. HRV acquired during two breathing protocols was applied, in order to limit respiratory bias. The role of sub-domains assessed within global cognition on the association was also examined.

Methods

Study design

Data from the first wave of The Irish Longitudinal Study on Ageing (TILDA) were analyzed. TILDA is a large prospective cohort study of ageing, comprised of community dwelling people aged 50 and over resident in the Republic of Ireland. A nationally representative sample was selected using the regularly updated RANSAM sampling technique [43]. Further detail of the study design is published elsewhere [14]. Data collected within TILDA are composed of three parts: (1) computer-assisted personal interviewing (CAPI), (2) self-completion questionnaire, and (3) a physical health assessment. Participants unwilling to undergo centre-based health assessment were offered an in-home health assessment, where a sub-group of measures was recorded. Electrocardiograms were conducted in the health centre assessment but not the home assessment. Ethical approval was obtained from Trinity College Dublin Ethics committee and all participants provided signed informed consent prior to the study. All 
experimental procedures adhered to the Declaration of Helsinki. This study uses data from the first wave of TILDA, collected between July 2009 and June 2011.

Cognitive assessment

Cognitive function was assessed in the TILDA study using a battery of cognitive tests [14]. This included the Montreal cognitive assessment (MOCA) [21], which was administered during the health assessment and used in the present study. MOCA is a measure of global cognitive function (score range 0-30), comprised of the sub-domains (1) memory (2) visuospatial function, (3) executive function, (4) sustained attention, (5) language, and (6) orientation. It was chosen for analysis over the mini mental state examination (MMSE) due to its higher predictive value for MCI [21] and less ceiling effect in a populationrepresentative sample.

\section{Measurement of RR interval variability}

Two 5 min supine resting surface 3-lead electrocardiograms (ECG) were recorded, during which subjects were instructed to breath spontaneously for the first period, and to control their breathing (paced) during the second period according to a pre-recorded set of auditory instructions [set at a rate of 12 cycles min-1 $(0.2 \mathrm{~Hz})]$. This protocol controlled experimentally for the effect of respiratory rate on spectral HRV indices. Recordings were obtained in a comfortably lit, quiet room at ambient temperature $\left(21-23^{\circ} \mathrm{C}\right)$ using digital recorders (Medilog Darwin ${ }^{\circ}$, Oxford Instruments Medical Ltd, UK).

The ECG was acquired and subsequently band-pass filtered $(0.01-1,000 \mathrm{~Hz})$. A proprietary algorithm was used to detect the $\mathrm{R}$ peak of each heart beat recorded on the ECG signal [24]. Its accuracy is described elsewhere [24]. Supraventricular ectopic beats and noise were excluded from the signal using linear interpolation. All recordings were screened for atrial fibrillation (AF) using criteria from the European society of cardiology (ESC) [4], and those identified with AF were subsequently excluded from analysis. Other arrhythmias were detected and excluded by the Darwin software system. Heart rate was recorded and controlled for in study analysis.

300-second epochs of RR interval data were analyzed. Statistical methods were used to derive the time domain index; the standard deviation of NN intervals (SDNN). Frequency domain (FD) features were calculated from spectral estimates derived using an autoregressive (Burg method) algorithm, with assignment of 256 discrete frequency bins. FD features were derived by integrating the power spectrum across bands using the fast Fourier transform (FFT) as recommended by the task force for HRV measurement [9]. Low frequency power (LF $0.04-0.15 \mathrm{~Hz}, \mathrm{~ms} 2$ ) and high frequency power (HF 0.15-0.4 $\mathrm{Hz}, \mathrm{ms} 2$ ) were calculated and expressed as a ratio LF:HF also.

A number of recordings $(n=424)$ were unsuitable for analysis of paced breathing data due to technical error or protocol non-compliance. These errors occurred completely at random and so were not thought to introduce a bias. Sensitivity analysis was performed to confirm this and restricted to cases with both spontaneous and paced breathing data only. Our results were not found to 
be sensitive to this potential bias. All subjects were therefore included in analysis of spontaneous breathing HRV data.

\section{Measurement of covariates}

Other measures acquired included age, gender, highest level of educational attainment (primary, secondary or tertiary), smoking status (never smoked, former or current), alcohol consumption (units weekly), body mass index (BMI) $(\mathrm{kg} / \mathrm{m} 2)$, total blood cholesterol ( $\mathrm{mmol} / \mathrm{L})$, and height $(\mathrm{cm})$. Physical activity was assessed using the International Physical Activity Questionnaire (IPAQ) short form, which classifies low, medium and high levels of activity. Systolic and diastolic blood pressures (SBP and DBP, respectively) were recorded during seated rest using a digital oscillometric blood pressure (BP) monitor $\left(\mathrm{OMRON}^{\mathrm{TM}}\right)$.

The Centre for Epidemiological Studies Depression (CES-D) scale was used with a cut-off score of 16 or above, to define subjects as depressed [28]. The Hospital Anxiety and Depression (HADS) scale was used to define subjects as having anxiety, using a cut-off criteria score of 11 or above [46]. This was obtained through a self-completion questionnaire. Self-reported cardiovascular diseases were documented and included: history of angina, myocardial infarction (MI), heart failure, diabetes mellitus (DM), stroke, transient ischaemic attack (TIA), and cardiac arrhythmias.

Medication use was recorded during the home interview (CAPI) and confirmed by cross-checking the medication labels. The anatomical therapeutic classification (ATC) codes were subsequently recorded for categorisation [44]. Medication categories adjusted for analysis were alpha-blockers ('CO2CA', 'CO2LE'), beta-blockers ('C07*'), calcium channel blockers ('CO8*'), cardiac glycosides ('C01A*'), midodrine ('C01CA'), anticholinergic agents ('N04A'), and anticholinesterase agents ('N06DA*, N07AA*').

\section{Statistical analysis}

Statistical analysis was performed using Stata version 12 (StataCorp, College Station, TX, USA). Distribution of continuous variables was assessed using Q-Q plots and histograms. Normally distributed variables were described as means and standard deviations (SD), and were compared across groups using independent $t$ tests. Non-normally distributed variables were described as medians and percentiles and compared using Mann-Whitney tests, and categorical variables were compared using Chi squared tests. HRV indices were divided into quintiles for analysis, to investigate the non-linear association between HRV and cognition. Quintiles for time and frequency domain indices were determined equally; by splitting the sample with non-missing data into fifths according to their index value. Multivariate linear regression was used to assess the relationship between HRV indices and MOCA score (continuous scale). Indices measured during spontaneous and paced breathing periods were compared. Significance was taken as $\mathrm{P}<0.05$. For each quintile increase in HRV, the standardized regression coefficient $(\beta)$ corresponded to the associated change in MOCA cognitive score. A core model (A) was first tested, adjusting for age, gender and education level, and a subsequent model (B) was tested, adjusting for these three variables, along with all remaining covariates described 
above, namely behavioral health (smoking, alcohol and physical activity), clinical profile (BMI, cholesterol, height, seated blood pressure), mental health (CES-D and HADS-A scores), cardiovascular disease (angina, MI, heart failure, DM, stroke, TIA, arrhythmias) and medications listed above. Adjusted predictive MOCA scores across quintiles of HRV indices were fitted.

Results

A total of 8,175 participants aged 50 years and older were recruited to the TILDA study [14]. Of these 5,036 agreed to undergo an in-centre health assessment, 4,763 of whom had technically adequate data for HRV analysis (see Fig. 1). A subset of 424 subjects had spontaneous breathing HRV data only.

http://static-content.springer.com/image/art\%3A10.1007\%2Fs10286-0130214-x/MediaObjects/10286_2013_214_Fig1_HTML.gif

Fig. 1

Flowchart of TILDA study participants eligible for ECG recording in TILDA

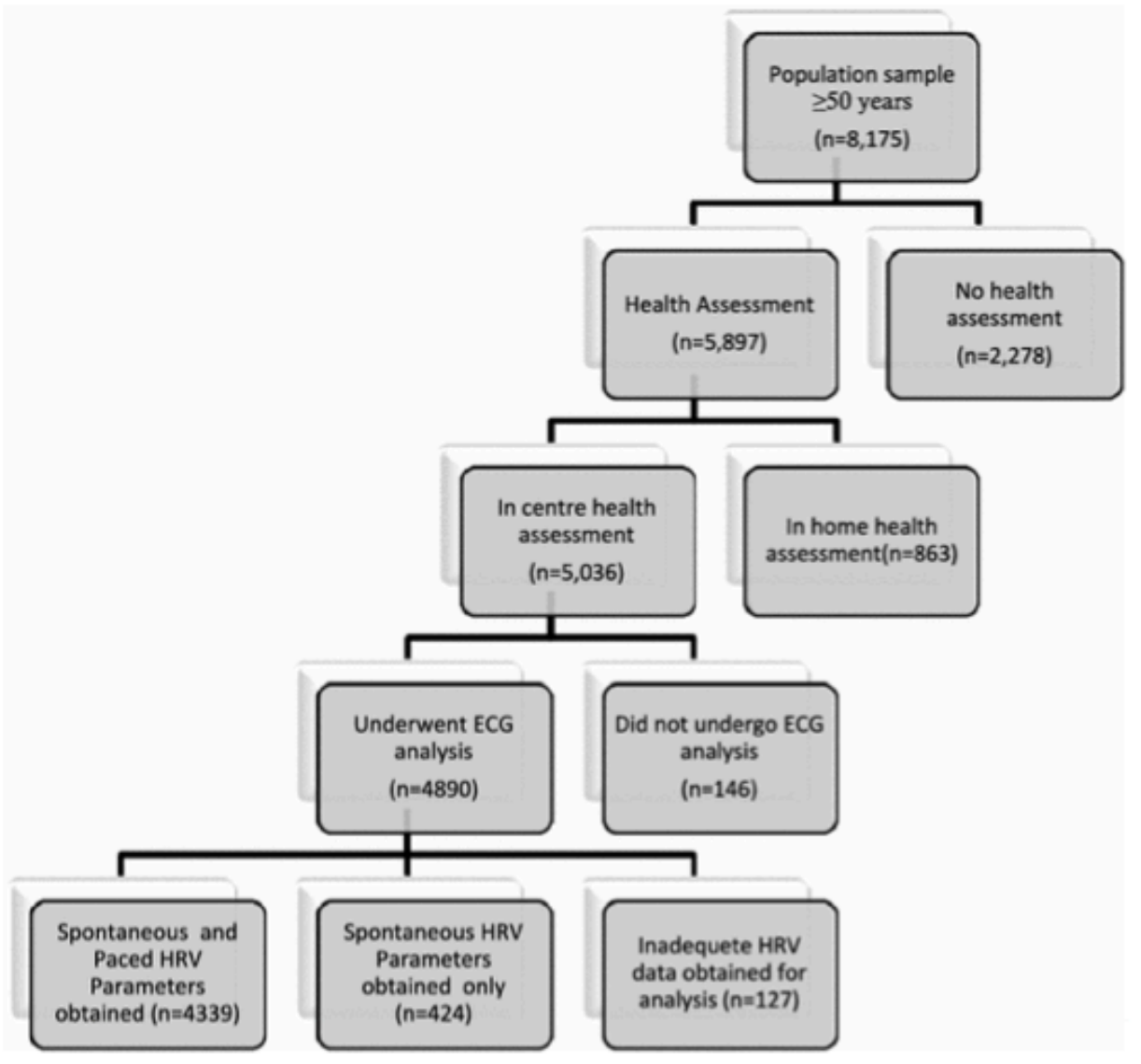

Fig. 1

Flowchart of TILDA study participants eligible for ECG recording in TILDA

The mean age was 61.7 and 2,618 (55 \%) were female. HRV patterns of the sample for both spontaneous and paced breathing periods are outlined in Table 1 , together with subject characteristics. Men had higher levels of alcohol consumption, former smoking, physical activity, BMI, SBP, DBP, and prevalence 
of cardiovascular disease. Women had higher levels of cholesterol and prevalence of anxiety and depression. Minimum, mean and maximum HR values were greater during paced breathing. HRV indices LF and HF were greater during spontaneous breathing, while SDNN and LF:HF ratio were greater during paced breathing period. 
Table 1

Characteristics of the stady posulation

\begin{tabular}{|c|c|c|c|}
\hline Characteristics & $\begin{array}{l}\operatorname{Mon} \\
(n=2145)\end{array}$ & $\begin{array}{l}\text { Wamen } \\
(n=2618)\end{array}$ & $\begin{array}{l}P \\
\text { value }\end{array}$ \\
\hline$A g e$, mana $=S D$ & $61.8 \sim 8.3$ & $61.5=8.3$ & 0.20 \\
\hline \multicolumn{4}{|l|}{ Eductution, $\%$ is } \\
\hline Peimary & 28.4 stes & $12.8 \mid 512)$ & \\
\hline Secorday & $41.5 \mathrm{pac}$ & $422(17,100)$ & 0.000 \\
\hline Tertiary & 35.1 pss) & 38.2 pow & 0.000 \\
\hline MOCA sedons" & $26(23,28 \mid$ & $28,24,26$ & 0.11 \\
\hline \multicolumn{4}{|l|}{ Smoking nan-s, $x$ m } \\
\hline Nonver & 38.3 |a21\% & $52.5(1,3754)$ & \\
\hline Former & 46.5 poes & 3248020 & 0.000 \\
\hline aumat & 15.2 م2e & $15.1(905)$ & 0.000 \\
\hline Standard thines ochsurted weebs & $4.5(0.6,12)$ & $1.5 \mathrm{p}, \mathrm{g}$ & 0.000 \\
\hline
\end{tabular}

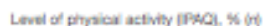

\begin{tabular}{|c|c|c|c|}
\hline Low & 28.1 Hasp & 31 sons & \\
\hline Mocium & $32.8 \mathrm{pas}$ & $30.5(1,005)$ & 0.0000 \\
\hline Hah & $42.3 p 42$ & $20.5(870)$ & 0.0001 \\
\hline 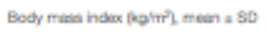 & $22.1 \times 4.4$ & $28+52$ & $<0.0001$ \\
\hline Toxa choustera ( & 4.9 a 1.1 & $5.4=1$ & $<0.0001$ \\
\hline 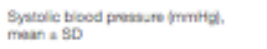 & $1900+18.3$ & 191 a 12.8 & $<0.0001$ \\
\hline 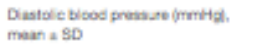 & $\Delta s: y+11$ & $81.2 \times 11.1$ & $<0.0001$ \\
\hline
\end{tabular}

Mertal twath $\%$ int

\begin{tabular}{|c|c|c|c|}
\hline Arsiety 0 ThDS $A=11$ & 15.3 p28 & $12.1(500)$ & $<0.5001$ \\
\hline Depression (CES- 0,16 ) & 7 (144) & $12(312)$ & $<0,0001$ \\
\hline
\end{tabular}

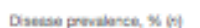

\begin{tabular}{|c|c|c|c|}
\hline Argin & $8(129)$ & $2.8(\mathrm{mp})$ & $<0,0001$ \\
\hline Myosardal intartion & $6.8(142)$ & $1.7(44)$ & $<0,0001$ \\
\hline Hean fulcre & $13(27)$ & $0.3 \%$ & $<0.0001$ \\
\hline Dutasons & 8.6 (tas) & $4.4(119)$ & $<0.0001$ \\
\hline Stsckem/7h & $2 . A(b)$ & 2.5 pes & 0.44 \\
\hline Carclac amplenta & 62 (193) & $5.5(145)$ & o.ss \\
\hline
\end{tabular}

\begin{tabular}{|c|c|c|c|}
\hline \multirow{2}{*}{ Heart rate varisbilty } & \multicolumn{2}{|l|}{$(n=4,339)$} & \multirow{2}{*}{$\begin{array}{l}P \\
\text { value }\end{array}$} \\
\hline & $\begin{array}{l}\text { Spontaneous brosthing } \\
\text { period }\end{array}$ & $\begin{array}{l}\text { Paoced broatting } \\
\text { period }\end{array}$ & \\
\hline $\begin{array}{l}\text { Mrimumitears nata } \\
\text { mman a SD }\end{array}$ & $578=2.8$ & $58.1=2.8$ & e.00301 \\
\hline $\begin{array}{l}\text { Mean tmat ruas, } \\
\text { mant } 2 \mathrm{SD}\end{array}$ & BA $=10.1$ & $66.4=10.8$ & $<00301$ \\
\hline $\begin{array}{l}\text { Maximum heart rate, } \\
\text { moan a SD }\end{array}$ & 75.76 .3 & $77.2 \times 152$ & $<0.0001$ \\
\hline SENNimap & $54.805 .5,47.3)$ & $41.1(90,55.7)$ & $<0.0001$ \\
\hline LF $\left(\mathrm{mr}^{2}\right)^{\mathrm{H}}$ & $164.4(04.1,268.8)$ & $148.3(74,4,2908)$ & $<00001$ \\
\hline $\mathrm{HF}\left(\mathrm{mr}^{2}\right)^{2}$ & $215.2(140.2,350.8)$ & $160.2(72.2,354.67$ & $<0.0001$ \\
\hline Lfirf natio & a.sa $(0.57,1.13)$ & $0.94 ; 0.52,1.68$ & $=00001$ \\
\hline
\end{tabular}

SD stantard deviron

"Summarized as modian (25th percensle, 75 h peroentile), because of skowed distroution 
The association between HRV (measured as quintiles) and MOCA score is summarized in Table 2, according to spontaneous or paced breathing. In Model A (adjusted for age, gender, and education), lower (quintiles of) LF:HF ratio during spontaneous breathing, lower HF and higher mean HR during paced breathing, and lower SDNN and LF during both periods were significantly associated with a lower MOCA score.

\section{Table 2}

Adjusted regression coefficients and $95 \%$ confidence intervals of cognitive performance comparing quintiles of $\mathrm{HRV}$ indices during spontaneous and paced breathing 


\begin{tabular}{|c|c|c|c|c|c|c|c|}
\hline \multicolumn{8}{|c|}{ 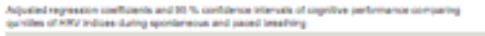 } \\
\hline \multirow{3}{*}{$\underset{ \pm}{\approx}$} & \multirow{3}{*}{$=$} & \multirow{3}{*}{ anem } & \multicolumn{5}{|c|}{ men- } \\
\hline & & & \multicolumn{3}{|c|}{ रित्रा } & \multicolumn{2}{|c|}{ 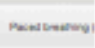 } \\
\hline & & & . & $\mathrm{Ex}^{\mathrm{N}}$ & . & , & ax \\
\hline$=$ & Nows & $\circ$ & & & 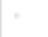 & & \\
\hline \multirow[t]{4}{*}{$\rightarrow$} & mess & 。 & sart & ât & $4 x$ & asu & A4 \\
\hline & eves & $\therefore$ & sut & ât & & $a x$ & 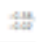 \\
\hline & 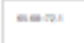 & a & siv & ats & & sar & atf \\
\hline & soxicos & 。 & сам & sto & & sail & 新 \\
\hline \multirow[t]{5}{*}{$\rightarrow$} & enses & a & & & . & & \\
\hline & exer & a & asser & $\therefore$ & $\omega$ & ase & sus \\
\hline & axan & $a$ & Ans & atio & & sau & st \\
\hline & exines & a & sus & 跲 & & swe & 年 \\
\hline & 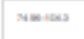 & a & sase & as & & case & sir \\
\hline on & wa & a & & & " & & 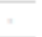 \\
\hline \multirow[t]{4}{*}{$\rightarrow$} & mases & a & outr & â" & $=$ & 2004 & ost \\
\hline & mee & a & om & 㚙 & & saus & ont \\
\hline & cases & a & oum & 哭 & & $2 \mathrm{as}$ & 盟 \\
\hline & atiey & a & ons & tan & & 204 & aty \\
\hline \multirow[t]{5}{*}{$\rightarrow$} & cast & a & . & . & . & . & . \\
\hline & 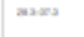 & a & $\operatorname{sen}$ & 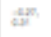 & $=$ & stum & 解 \\
\hline & weses & $\therefore$ & sem & 浣 & & nas & 是 \\
\hline & cones & 。 & sue & An & & asu & sis \\
\hline & cenes & . & 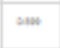 & 嵒 & & sout & 解 \\
\hline 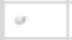 & $=x$ & 。 & . & . & & & \\
\hline \multirow[t]{4}{*}{-} & atie & $\approx$ & san & $a^{n a}$ & $-1=$ & $2 \mathrm{ex}$ & औौำ \\
\hline & nesory & * & surt & 㞫 & & saw & ont \\
\hline & I & a & om & 㚙 & & 200 & 에 \\
\hline & $=2.002$ & a & sav" & 늠 & & $=4$ & ats \\
\hline \multirow[t]{5}{*}{$\rightarrow$} & neas & a & & & . & & \\
\hline & стия & a & onr & $\Delta$ & $=$ & w4 & an \\
\hline & 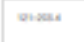 & a & sall & 㠩 & & new & on \\
\hline & $m=x$ & a & sim & 跑 & & 200 & 品 \\
\hline & mase & a & satu & 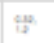 & & sear & par \\
\hline 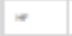 & coss & $a$ & & & 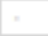 & & \\
\hline \multirow[t]{4}{*}{$\rightarrow$} & - & a & 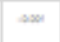 & (4) & $\omega$ & sau & 器 \\
\hline & maser & $a$ & can & مئ & & $w$ & 연 \\
\hline & 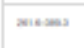 & a & sin & A & & uas & 罥 \\
\hline & wamas & a & cosest & 해해 & & sau & 魷 \\
\hline \multirow[t]{5}{*}{$\rightarrow$} & neses & $\approx$ & & & & & \\
\hline & exisen & $\approx$ & $\sin$ & at & $\omega$ & 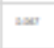 & At \\
\hline & wises & 。 & sserr & 虽 & & as & 형 \\
\hline & $\operatorname{sen} x$ & $\approx$ & sats & 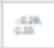 & & เй & Astr \\
\hline & $-\infty y$ & 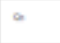 & one & A & & asm & ồ \\
\hline$\cdots$ & сове & a & & & & . & \\
\hline- & ина & 4 & sin & 竞 & - & tom & and \\
\hline \multirow[t]{3}{*}{$\rightarrow \wedge$} & is & a & su & ax & & $4=$ & and \\
\hline & ... & a & onem & 然 & & сам & sat \\
\hline & (tans. & o. & onim & 紿 & & sw & 台保 \\
\hline \multirow[t]{5}{*}{$\rightarrow$} & |wese & $a$ & & & & . & \\
\hline & aten & a & жั & at & -4 & sain & 今 \\
\hline & : & a & ${ }^{n}$ & ats & & 4"w & A4 \\
\hline & $1=+\infty$ & 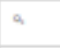 & 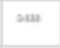 & 象 & & сан & \% \\
\hline & Iatse & a & cous & 48 & & west & 剑 \\
\hline
\end{tabular}


Model A included age, gender and education

Model B included age (both linear and non-linear effects), gender, education, smoking status, alcohol consumption, exercise level, body mass index, total cholesterol level, height, systolic blood pressure, diastolic blood pressure, CES-D depression score, HADS-A anxiety score, angina, myocardial infarction, heart failure, diabetes mellitus, stroke/transient ischaemic attack, cardiac arrhythmias, mean heart rate, and the following medications: alpha-blockers, beta-blockers, calcium channel blockers, midodrine, anti-cholinergics, and anti-cholinesterases

Statistically significant relationships are in boldface

HR heart rate, SDNN standard deviation of NN intervals, LF low frequency, HF high frequency, $\beta$ standardized regression coefficients

Following further adjustment for other confounders of cardiovascular and cognitive health (Model B), lower HRV remained significantly associated with lower MOCA score across several indices. Lower (quintiles of) SDNN, LF and LF:HF ratio during both periods were associated with a lower MOCA score. The association between higher mean HR and lower MOCA score remained significant in Model B, during paced breathing only.

There was a dose response in the multivariate adjusted effect size between HRV and MOCA score, for the indices LF and LF:HF ratio. MOCA scores were fitted across quintiles of paced LF, with adjustment for covariates, to illustrate this dose response (see Fig. 2). Subjects in the lowest LF quintile group had an adjusted estimated MOCA score of 25 (95\% CI; 24.8-25.3), whereas subjects in the highest LF quintile group scored 25.7 (95 \% CI; 25.5-26).

http://static-content.springer.com/image/art\%3A10.1007\%2Fs10286-0130214-x/MediaObjects/10286_2013_214_Fig2_HTML.gif

Fig. 2

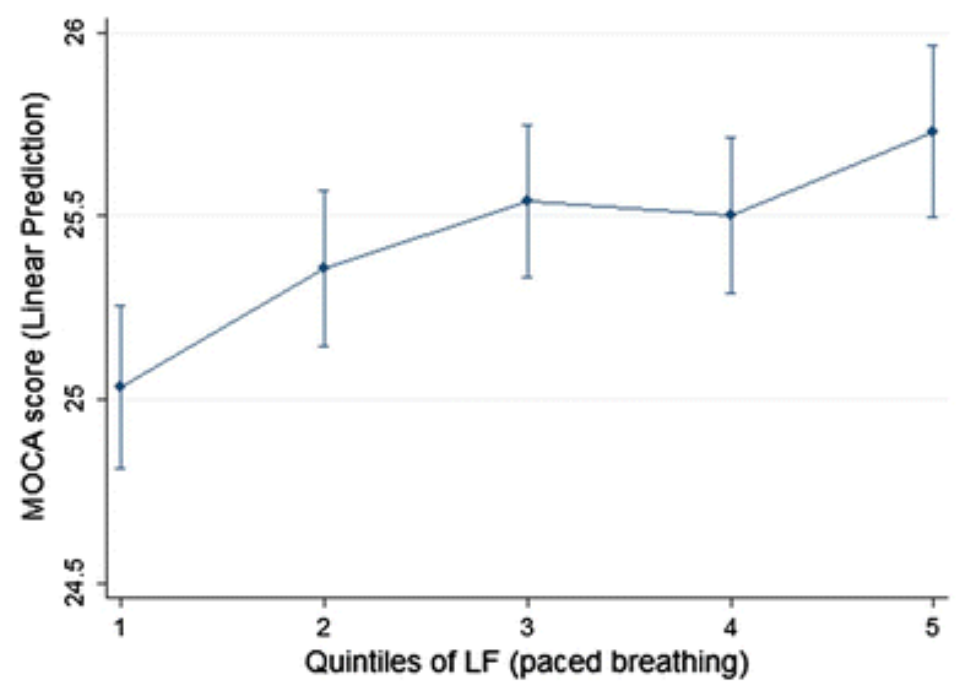


Fitted MOCA scores across quintiles of Low Frequency HRV with 95 \% CIs. Analysis adjusted for age (both linear and non-linear effects), gender, education, smoking status, alcohol consumption, exercise level, body mass index, total cholesterol level, height, systolic blood pressure, diastolic blood pressure, CES-D depression score, HADS-A anxiety score, angina, myocardial infarction, heart failure, diabetes mellitus, stroke/transient ischaemic attack, cardiac arrhythmias, mean heart rate, and the following medications: alpha-blockers, beta-blockers, calcium channel blockers, midodrine, anti-cholinergics, and anti-cholinesterases. MOCA montreal cognitive assessment, HRV heart rate variability, LF low frequency

Figure 3 illustrates the HRV changes associated with poor cognitive performance. SDNN2 is shown instead of SDNN as it is indicative of total power and yielded identical significance to SDNN in analysis. Regression analysis using a dichotomised MOCA score, according to the criteria for cognitive impairment (scores below 24) [7, 17], yielded broadly similar results (not shown). Further sub-analysis stratifying by gender yielded similar results between men and women.

Fig. 3

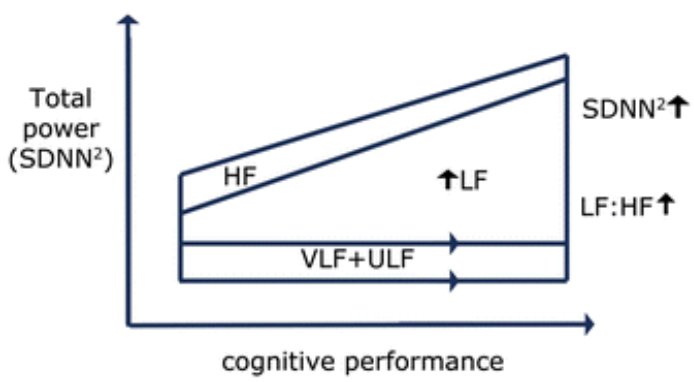

Schema explaining the HRV changes independently associated with cognitive decline. SDNN standard deviation of NN intervals, ULF ultra low frequency, VLF very low frequency, LF low frequency, HF high frequency

Analysis of the sub-domains of MOCA score was performed to determine the component responsible for the effect of HRV on cognitive function. Confounders included in Model B above were adjusted for here (see Table 3). Domains of memory recall and language predominantly accounted for the association between HRV and MOCA score.

Table 3

Model B regression coefficients and standard errors of MOCA sub-domains, comparing 1st to 5 th quintiles of HRV indices 


\begin{tabular}{|c|c|c|c|c|c|c|c|c|}
\hline \multicolumn{9}{|c|}{$\begin{array}{l}\text { Model B regression coefficients and standard errors of MOCA sub-domains, comparing 1st to } 5 \text { th quintiles } \\
\text { of HRV indices }\end{array}$} \\
\hline \multirow{3}{*}{$\begin{array}{l}\text { MOCA } \\
\text { subdomain }\end{array}$} & \multirow{3}{*}{$\begin{array}{l}\text { Breathing } \\
\text { period }\end{array}$} & \multicolumn{2}{|c|}{ Mean HR } & \multicolumn{2}{|c|}{ SDNN } & \multicolumn{2}{|l|}{ LF } & \multirow{2}{*}{$\begin{array}{l}\mathrm{HF} \\
\mathrm{Q}_{\varepsilon}\end{array}$} \\
\hline & & \multicolumn{2}{|l|}{$\mathrm{Q}_{5}$} & \multicolumn{2}{|l|}{$Q_{5}$} & \multicolumn{2}{|l|}{$Q_{5}$} & \\
\hline & & $\beta$ & SE & $\beta$ & SE & $\beta$ & SE & $\beta$ \\
\hline \multirow{2}{*}{$\begin{array}{l}\text { Memory } \\
\text { recall }\end{array}$} & Spont ${ }^{\mathrm{a}}$ & -0.086 & 0.078 & 0.249 & 0.084 & 0.435 & 0.084 & 0.1 \\
\hline & Paced & -0.154 & 0.082 & 0.273 & 0.09 & 0.335 & 0.085 & $0 . c$ \\
\hline \multirow{2}{*}{$\begin{array}{l}\text { Visuospatial } \\
\text { function }\end{array}$} & Spont ${ }^{\mathrm{a}}$ & -0.064 & 0.039 & 0.108 & 0.043 & 0.085 & 0.043 & $0 . c$ \\
\hline & Paced & -0.125 & 0.042 & -0.02 & 0.046 & 0.073 & 0.043 & $0 . c$ \\
\hline \multirow{2}{*}{$\begin{array}{l}\text { Executive } \\
\text { function }\end{array}$} & Spont ${ }^{\mathrm{a}}$ & -0.035 & 0.048 & 0.057 & 0.052 & 0.051 & 0.052 & -0 \\
\hline & Paced & -0.139 & 0.051 & 0.026 & 0.056 & 0.041 & 0.053 & $0 . c$ \\
\hline \multirow{2}{*}{$\begin{array}{l}\text { Sustained } \\
\text { attention }\end{array}$} & Spont ${ }^{\mathrm{a}}$ & -0.071 & 0.043 & 0.079 & 0.047 & 0.102 & 0.047 & -0 \\
\hline & Paced & -0.097 & 0.046 & 0.058 & 0.05 & 0.103 & 0.048 & $0 . c$ \\
\hline \multirow[t]{2}{*}{ Language } & Spont ${ }^{\mathrm{a}}$ & -0.026 & 0.059 & 0.166 & 0.063 & 0.184 & 0.063 & $0 . c$ \\
\hline & Paced & -0.163 & 0.062 & 0.153 & 0.068 & 0.167 & 0.065 & $0 . c$ \\
\hline \multirow[t]{2}{*}{ Orientation } & Spont ${ }^{\mathrm{a}}$ & -0.019 & 0.015 & 0.02 & 0.016 & 0.012 & 0.016 & -0 \\
\hline & Paced & -0.016 & 0.016 & 0.019 & 0.018 & 0.017 & 0.017 & $0 . c$ \\
\hline
\end{tabular}

Model included age (both linear and non-linear effects), gender, education, smoking status, alcohol consumption, exercise level, body mass index, total cholesterol level, height, systolic blood pressure, diastolic blood pressure, CES-D depression score, HADS-A anxiety score, angina, myocardial infarction, heart failure, diabetes mellitus, stroke/transient ischaemic attack, cardiac murmurs, cardiac arrhythmias, mean heart rate, and the following medications: alphablockers, beta-blockers, calcium channel blockers, midodrine, anti-cholinergics, and anti-cholinesterases. Paced $n=4,339$

Statistically significant relationships are in boldface (ANOVA across all quintiles) $\beta$ standardized regression coefficients

aSpont $=$ spontaneous $(n=4,863)$

Discussion

HRV was independently associated with poor global cognitive performance in a large representative population sample of community dwelling, non-demented persons over 50 years. Furthermore, there was a graded association between 
degrees of impaired cognitive performance and spectral indices of LF and LF:HF ratio, which was independent of respiration, indicating impaired baroreflex function and sympathovagal homeostasis in early cognitive ageing. This is the first such study in a population sample of both men and women detailed with information on co-medications and co-morbidities. Memory recall and language were most strongly associated with lower HRV values, suggesting implication of a common neuro-anatomical pathway, possibly the fornix, which is the predominant outflow tract of the hippocampus and functions in autonomic control [6]. The direction of causality will be explored in futures waves of this longitudinal study.

The interpretation of LF as an index of sympathetic function is debated, with recent evidence suggesting $L F$ to be more reflective of baroreflex function than sympathetic function $[1,20]$. LF:HF ratio is considered a measure of sympathovagal balance, however, it may be more closely associated with cardiac autonomic modulation, a term more inclusive of complexities of the autonomic system [22]. In light of this, findings from this study suggest that reduced overall HRV (SDNN), baroreflex function (LF), and cardiac autonomic modulation (LF:HF) are all associated with poor cognitive performance in the community dwelling population. The methods and details applied in TILDA differ from previous studies which have examined this association. Kim et al. [15] reported an association between low LF and HF and poor global cognition in a convenience sample of 311 female participants. In the Whitehall study, which was a younger cohort of civil servants, no association was noted either crosssectionally or longitudinally [3]. A possible explanation for this difference is social class.

The MOCA sub-domains of memory recall and language were most strongly associated with lower HRV values. This is in accordance with previous studies reporting significant associations between HRV and verbal memory recall [35]. The integrity of the fornix, which is the predominant outflow tract of the hippocampus, is predictive of memory performance and progression from MCI to $\mathrm{AD}$ [19]. This region is also implicated in autonomic control [6], suggesting that the fornix may form the anatomical location underlying the relationship between HRV and cognition.

The cholinergic anti-inflammatory pathway may also explain the association between reduced HRV and cognitive decline. Efferent vagal nerve activity inhibits pro-inflammatory cytokine release, protecting against systemic inflammation [25]. Reduced LF, HF, and SDNN along with increased HR are associated with higher levels of C-reactive protein (CRP) $[18,31,38]$ and IL-6 [38], both of which are associated with cognitive decline, thus inflammation may mediate the relationship between reduced HRV and cognitive performance [34, $40,41]$.

Another hypothesis implicates Noradrenaline (NAd). Up-regulation of NAd is proposed to optimize function of the key elements of cognitive reserve-namely educational level, IQ, mental and social engagement-which together lengthen the period between onset of pathological changes consistent with $\mathrm{AD}$ and 
symptoms of the disease (Robertson [30]). Reduction in NAd and acetylcholine (Ach) function (neurotransmitters of the SNS) results in lower HRV values.

Hypertension is an established predictor of cognitive decline [10], and is also associated with reduced HRV [37], indicating a possible role in the relationship of HRV and cognition. We controlled for resting blood pressure, however, demonstrating that hypertension does not account for our findings.

Our analysis adjusted for both linear and non-linear effects of chronological age; however, the role of unidentified biological ageing processes cannot be excluded. Hence, such factors may account for decline in both HRV and cognitive function, without the two systems being causally related.

A standardized protocol for recording HRV describing the optimal duration, environment, and respiratory rate for recording has yet to be established. Adherence to procedure recommendations [9] remains sub-standard, limiting the interpretive value of absolute HRV data between studies [23]. Re-test reliability of HRV measures is poor [33], however, paced breathing data collection protocol improves reliability and so its use is encouraged [26, 32]. For this reason, a paced breathing protocol was implemented here. The association between HRV and cognitive function, adjusted for respiration, indicates that a stronger relationship possibly exists than previously reported, as studies to date have recorded HRV during spontaneous breathing only. Given the practicality of delivering comprehensive assessments to participants nationwide it was not possible to ask participants to fast or to standardize The time of the day that health assessments were performed. The time of the day, however, was recorded and subsequently analyzed to determine its effect on HRV. It was not independently associated with HRV indices.

The similarity of results when re-analysis was performed using dichotomous MOCA score $(<24)$ serves to further validate our findings of an association between reduced HRV and cognitive decline (not shown).

The study sample is nationally representative of the community dwelling population aged 50 years and older living in Ireland. For this reason, our results reflect all members of the community and sub-group analyses are valid. TILDA uniquely measures HRV, global and specific cognitive function assessments, and is coupled with specific details on medications. This allows for correction of possible medication confounding effects on HRV.

\section{Conclusion}

In light of the ageing population demographic predicted in the coming years, interventional strategies to combat the associated rise in burden of cognitive disorders is imperative. This study has for the first time reported an association between HRV (as a measure of cardiac autonomic function) and global cognitive performance at a population level. Reduced overall HRV (SDNN), baroreflex function (LF), and cardiac autonomic modulation (LF:HF) are all independently associated with reduced cognitive performance. This may reflect shared anatomical pathways or mechanistic pathways such as inflammation or 
depletion of NAd reserves. Longitudinal follow-up will help to explain our observations.

Acknowledgments

Financial contributions from the following entities would like to be acknowledged; The Atlantic Philanthropies (research grant to the Irish Longitudinal Study of Ageing); Irish Life plc and the Irish Government (grant to the Irish Longitudinal Study of Ageing). We would also like to acknowledge the participants in the study, and the studies nurses and administrators.

Conflict of interest

None declared.

References

1.

Baumert M, Lambert GW, Dawood T, Lambert EA, Esler MD, McGrane M, Barton D, Nalivaiko E (2008) QT interval variability and cardiac norepinephrine spillover in patients with depression and panic disorder. Am J Physiol Heart circulatory physiol 295(3):H962-H968. doi:10.1152/ajpheart.00301.2008 .

2.

Bischkopf J, Busse A, Angermeyer MC (2002) Mild cognitive impairment-a review of prevalence, incidence and outcome according to current approaches. Acta Psychiatr Scand 106(6):403-414,.

3.

Britton A, Singh-Manoux A, Hnatkova K, Malik M, Marmot MG, Shipley M (2008) The association between heart rate variability and cognitive impairment in middle-aged men and women. The Whitehall II cohort study. Neuroepidemiology 31(2):115-121. doi:10.1159/000148257, .

4.

Camm AJ, Kirchhof P, Lip GYH, Schotten U, Savelieva I, Ernst S, Van Gelder IC, AlAttar N, Hindricks G, Prendergast B (2010) Guidelines for the management of atrial fibrillation: the task force for the management of atrial fibrillation of the European society of cardiology (ESC). Europace 12(10):1360-1420,.

\section{5.}

Carnethon MR, Golden SH, Folsom AR, Haskell W, Liao D (2003) Prospective investigation of autonomic nervous system function and the development of type 2 diabetes: the atherosclerosis risk in communities study, 1987-1998. Circulation 107(17):2190-2195. doi:10.1161/01.cir.0000066324.74807.95 ,.

6. 
Castle M, Comoli E, Loewy AD (2005) Autonomic brainstem nuclei are linked to the hippocampus. Neuroscience 134(2):657-669. doi:10.1016/j.neuroscience. 2005.04.031,

\section{7.}

Coen RF, Cahill R, Lawlor BA (2011) Things to watch out for when using the Montreal cognitive assessment (MoCA). Int J Geriatr Psychiatry 26(1):107-108. doi:10.1002/gps.2471,

8.

Cui Y, Liu B, Luo S, Zhen X, Fan M, Liu T, Zhu W, Park M, Jiang T, Jin JS, The Alzheimer's Disease Neuroimaging, I (2011) Identification of conversion from mild cognitive impairment to Alzheimer's disease using multivariate predictors. PLoS ONE 6(7):e21896. doi:10.1371/journal.pone.0021896 ,.

9.

Electrophysiology, T.F.o.t.E.S.o.C.t.N.A.S.o.P. (1996) Heart rate variability: standards of measurement, physiological interpretation, and clinical use. Circulation 93(5):1043-1065. doi:10.1161/01.cir.93.5.1043 .

10.

Elias MF, Goodell AL, Dore GA (2012) Hypertension and cognitive functioning: a perspective in historical context. Hypertension 60(2):260-268,.

\section{1.}

Ewers M, Walsh C, Trojanowski JQ, Shaw LM, Petersen RC, Jack CR Jr, Feldman HH, Bokde AL, Alexander GE, Scheltens P, Vellas B, Dubois B, Weiner M, Hampel $\mathrm{H}$ (2012) Prediction of conversion from mild cognitive impairment to Alzheimer's disease dementia based upon biomarkers and neuropsychological test performance. Neurobiol Aging 33(7):1203-1214. doi:10.1016/j. neurobiolaging.2010.10.019,

\section{2.}

Felber Dietrich D, Schindler C, Schwartz J, Barthelemy JC, Tschopp JM, Roche F, von Eckardstein A, Brandli O, Leuenberger P, Gold DR, Gaspoz JM, AckermannLiebrich U (2006) Heart rate variability in an ageing population and its association with lifestyle and cardiovascular risk factors: results of the SAPALDIA study. Europace 8(7):521-529. doi:10.1093/europace/eul063 ,.

13.

Hollingworth P, Harold D, Sims R, Gerrish A, Lambert JC, Carrasquillo MM, Abraham R, Hamshere ML, Pahwa JS, Moskvina V, Dowzell K, Jones N, Stretton A, Thomas C, Richards A, Ivanov D, Widdowson C, Chapman J, Lovestone S, Powell J, Proitsi P, Lupton MK, Brayne C, Rubinsztein DC, Gill M, Lawlor B, Lynch A, Brown KS, Passmore PA, Craig D, McGuinness B, Todd S, Holmes C, Mann D, Smith AD, Beaumont H, Warden D, Wilcock G, Love S, Kehoe PG, Hooper NM, Vardy ER, 
Hardy J, Mead S, Fox NC, Rossor M, Collinge J, Maier W, Jessen F, Ruther E, Schurmann B, Heun R, Kolsch H, van den Bussche H, Heuser I, Kornhuber J, Wiltfang J, Dichgans M, Frolich L, Hampel H, Gallacher J, Hull M, Rujescu D, Giegling I, Goate AM, Kauwe JS, Cruchaga C, Nowotny P, Morris JC, Mayo K, Sleegers K, Bettens K, Engelborghs S, De Deyn PP, Van Broeckhoven C, Livingston G, Bass NJ, Gurling H, McQuillin A, Gwilliam R, Deloukas P, Al-Chalabi A, Shaw CE, Tsolaki M, Singleton AB, Guerreiro R, Muhleisen TW, Nothen MM, Moebus S, Jockel KH, Klopp N, Wichmann HE, Pankratz VS, Sando SB, Aasly JO, Barcikowska M, Wszolek ZK, Dickson DW, Graff-Radford NR, Petersen RC, van Duijn CM, Breteler MM, Ikram MA, DeStefano AL, Fitzpatrick AL, Lopez O, Launer LJ, Seshadri S, Berr C, Campion D, Epelbaum J, Dartigues JF, Tzourio C, Alperovitch A, Lathrop M, Feulner TM, Friedrich P, Riehle C, Krawczak M, Schreiber S, Mayhaus M, Nicolhaus S, Wagenpfeil S, Steinberg S, Stefansson H, Stefansson K, Snaedal J, Bjornsson S, Jonsson PV, Chouraki V, Genier-Boley B, Hiltunen M, Soininen H, Combarros O, Zelenika D, Delepine M, Bullido MJ, Pasquier F, Mateo I, FrankGarcia A, Porcellini E, Hanon O, Coto E, Alvarez V, Bosco P, Siciliano G, Mancuso M, Panza F, Solfrizzi V, Nacmias B, Sorbi S, Bossu P, Piccardi P, Arosio B, Annoni G, Seripa D, Pilotto A, Scarpini E, Galimberti D, Brice A, Hannequin D, Licastro F, Jones L, Holmans PA, Jonsson T, Riemenschneider M, Morgan K, Younkin SG, Owen MJ, O'Donovan M, Amouyel P, Williams J (2011) Common variants at ABCA7, MS4A6A/MS4A4E, EPHA1, CD33 and CD2AP are associated with Alzheimer's disease. Nat Genet 43(5):429-435. doi:10.1038/ng.803 ,.

\section{4.}

Kearney PM, Cronin H, O’Regan C, Kamiya Y, Savva GM, Whelan B, Kenny R (2011) Cohort profile: the Irish longitudinal study on ageing. Int J Epidemiol 40(4):877-884. doi:10.1093/ije/dyr116, .

15.

Kim DH, Lipsitz LA, Ferrucci L, Varadhan R, Guralnik JM, Carlson MC, Fleisher LA, Fried LP, Chaves PH (2006) Association between reduced heart rate variability and cognitive impairment in older disabled women in the community: women's Health and Aging Study I. J Am Geriatr Soc 54(11):1751-1757. doi:10.1111/j. 1532-5415.2006.00940.x,

\section{6.}

Kim JW, Lee DY, Lee BC, Jung MH, Kim H, Choi YS, Choi IG (2012) Alcohol and cognition in the elderly: a review. Psychiatry Investigation 9(1):8-16. doi:10. 4306/pi.2012.9.1.8,

\section{7.}

Lee JY, Dong Woo L, Cho SJ, Na DL, Hong Jin J, Kim SK, You Ra L, Youn JH, Kwon M, Lee JH, Maeng Je C (2008) Brief screening for mild cognitive impairment in elderly outpatient clinic: validation of the Korean version of the montreal cognitive assessment. J Geriatr Psychiatry Neurol 21(2):104-110. doi:10.1177/ 0891988708316855 , 
Li X, Shaffer ML, Rodriguez-Colon SM, He F, Bixler EO, Vgontzas AN, Wolbrette DL, Wu C, Ball RW, Liao D (2011) Systemic inflammation and circadian rhythm of cardiac autonomic modulation. Autonomic Neurosci: Basic Clin 162(1-2):72-76. doi:10.1016/j.autneu.2011.03.002 .

19.

Mielke MM, Okonkwo OC, Oishi K, Mori S, Tighe S, Miller MI, Ceritoglu C, Brown T, Albert M, Lyketsos CG (2012) Fornix integrity and hippocampal volume predict memory decline and progression to Alzheimer's disease. Alzheimer's Dementia: J Alzheimer's Assoc 8(2):105-113. doi:10.1016/j.jalz.2011.05.2416.

20.

Moak JP, Goldstein DS, Eldadah BA, Saleem A, Holmes C, Pechnik S, Sharabi Y (2009) Supine low-frequency power of heart rate variability reflects baroreflex function, not cardiac sympathetic innervation. Clevel Clin J Med 76(Suppl 2):S51-S59. doi:10.3949/ccjm.76.s2.11.

\section{1.}

Nasreddine ZS, Phillips NA, Bedirian V, Charbonneau S, Whitehead V, Collin I, Cummings JL, Chertkow H (2005) The montreal cognitive assessment, MoCA: a brief screening tool for mild cognitive impairment. J Am Geriatr Soc 53(4):695699. doi:10.1111/j.1532-5415.2005.53221.x ,.

22.

Nicolini P, Ciulla MM, De Asmundis C, Magrini F, Brugada P (2012) The prognostic value of heart rate variability in the elderly, changing the perspective: from sympathovagal balance to chaos theory. Pacing Clin Electrophysiol 35(5):622-638. doi:10.1111/j.1540-8159.2012.03335.x ,.

23.

Nunan D, Sandercock GR, Brodie DA (2010) A quantitative systematic review of normal values for short-term heart rate variability in healthy adults. Pacing Clin Electrophysiol 33(11):1407-1417. doi:10.1111/j.1540-8159.2010.02841.x ,.

24.

Pardey J, Jouravleva S (2004) The next-generation holter revolution: from analyse-edit-print to analyse-print. Comput Cardiol 2004:373-376

25.

Pavlov VA, Tracey KJ (2005) The cholinergic anti-inflammatory pathway. Brain Behav Immun 19(6):493-499. doi:10.1016/j.bbi.2005.03.015 ,.

26.

Pinna GD, Maestri R, Torunski A, Danilowicz-Szymanowicz L, Szwoch M, La Rovere MT, Raczak G (2007) Heart rate variability measures: a fresh look at reliability. Clin Sci (Lond) 113(3):131-140. 
Qiu C, Winblad B, Viitanen M, Fratiglioni L (2003) Pulse pressure and risk of Alzheimer disease in persons aged 75 years and older: a community-based, longitudinal study. Stroke 34(3):594-599. doi:10.1161/01.str.0000060127. 96986.f4, .

28.

Radloff LS (1977) The CES-D Scale. Appl Psychol Meas 1(3):385-401. doi:10. $1177 / 014662167700100306$.

29.

Robertson IH (2013) A noradrenergic theory of cognitive reserve: implications for Alzheimer's disease. Neurobiol Aging 34(1):298-308. doi:10.1016/j. neurobiolaging.2012.05.019

30.

Rountree S, Waring S, Chan W, Lupo P, Darby E, Doody R (2007) Importance of subtle amnestic and nonamnestic deficits in mild cognitive impairment: prognosis and conversion to dementia. Dement Geriatr Cogn Disord 24(6):476482 .

31.

Sajadieh A, Nielsen OW, Rasmussen V, Hein HO, Abedini S, Hansen JF (2004) Increased heart rate and reduced heart-rate variability are associated with subclinical inflammation in middle-aged and elderly subjects with no apparent heart disease. Eur Heart J 25(5):363-370. doi:10.1016/j.ehj.2003.12.003 ,.

32.

Sandercock G, Gladwell V, Dawson S, Nunan D, Brodie D, Beneke R (2008) Association between RR interval and high-frequency heart rate variability acquired during short-term, resting recordings with free and paced breathing. Physiol Meas 29(7):795-802. doi:10.1088/0967-3334/29/7/008 ,.

33.

Sandercock GRH, Bromley PD, Brodie DA (2005) The reliability of short-term measurements of heart rate variability. Int J Cardiol 103(3):238-247,.

34.

Schram MT, Euser SM, De Craen AJM, Witteman JC, Frolich M, Hofman A, Jolles J, Breteler MMB, Westendorp RGJ (2007) Systemic markers of inflammation and cognitive decline in old age. J Am Geriatr Soc 55(5):708-716,.

35.

Shah AJ, Su S, Veledar E, Bremner JD, Goldstein FC, Lampert R, Goldberg J, Vaccarino $V$ (2011) Is heart rate variability related to memory performance in 
middle-aged men? Psychosom Med 73(6):475-482. doi:10.1097/PSY. 0b013e3182227d6a, .

36.

Simone MJ, Tan ZS (2011) The role of inflammation in the pathogenesis of delirium and dementia in older adults: a review. CNS Neurosci Ther 17(5):506513 .

37.

Singh JP, Larson MG, Tsuji H, Evans JC, O’Donnell CJ, Levy D (1998) Reduced heart rate variability and new-onset hypertension: insights into pathogenesis of hypertension: the Framingham Heart Study. Hypertension 32(2):293-297,.

38.

Sloan RP, McCreath H, Tracey KJ, Sidney S, Liu K, Seeman T (2007) RR interval variability is inversely related to inflammatory markers: the CARDIA study. Molecular Med (Cambridge, Mass) 13(3-4):178-184. doi:10.2119/2006-00112. Sloan

39.

Tan ZS, Beiser AS, Fox CS, Au R, Himali JJ, Debette S, Decarli C, Vasan RS, Wolf PA, Seshadri S (2011) Association of metabolic dysregulation with volumetric brain magnetic resonance imaging and cognitive markers of subclinical brain aging in middle-aged adults: the Framingham Offspring Study. Diabetes Care 34(8):17661770. doi:10.2337/dc11-0308,.

40.

Trollor JN, Smith E, Agars E, Kuan SA, Baune BT, Campbell L, Samaras K, Crawford J, Lux O, Kochan NA, Brodaty H, Sachdev P (2012) The association between systemic inflammation and cognitive performance in the elderly: the Sydney Memory and Ageing Study. Age 34(5):1295-1308,.

41.

van den Kommer TN, Dik MG, Comijs HC, Jonker C, Deeg DJH (2012) The role of lipoproteins and inflammation in cognitive decline: Do they interact? Neurobiol Aging 33(1):196.e1-196.e12.

42.

van Rossum IA, Vos S, Handels R, Visser PJ (2010) Biomarkers as predictors for conversion from mild cognitive impairment to Alzheimer-type dementia: implications for trial design. J Alzheimers Dis 20(3):881-891. doi:10.3233/jad2010-091606,

43.

Whelan BJ (1979) RANSAM: A random sampling design for Ireland. Econ Soc Rev 10(2):169-175 
44.

WHO Collaborating Centre for Drug Statistics Methodology (2011) Guidelines for ATC classification and DDD assignment 2012. Oslo

45.

Wolf PA (2012) Contributions of the framingham heart study to stroke and dementia epidemiologic research at 60 years. Arch Neurol 69(5):567-571. doi:10.1001/archneurol.2011.977 ,.

46.

Zigmond AS, Snaith R (1983) The hospital anxiety and depression scale. Acta Psychiatr Scand 67(6):361-370,. 